\title{
Spacecraft Fire Detection: Smoke Properties and Transport in Low-Gravity
}

\author{
David L. Urban ${ }^{1}$, Gary A. Ruff ${ }^{2}$ and John E. Brooker ${ }^{3}$, \\ NASA Glenn Research Center, Cleveland, Ohio 44135 \\ Thomas Cleary ${ }^{4}$ and Jiann Yang ${ }^{5}$ \\ National Institute of Standards and Technology, Gaithersburg, Maryland 20899 \\ George Mulholland ${ }^{6}$ \\ The University of Maryland, College Park, Maryland 20742 \\ and \\ Zeng-guang Yuan ${ }^{7}$ \\ National Center for Space Exploration Research, Cleveland, Ohio 44135
}

\begin{abstract}
Results from a recent smoke particle size measurement experiment conducted on the International Space Station (ISS) are presented along with the results from a model of the transport of smoke in the ISS. The experimental results show that, for the materials tested, a substantial portion of the smoke particles are below $500 \mathrm{~nm}$ in diameter. The smoke transport model demonstrated that mixing dominates the smoke transport and that consequently detection times are longer than in normal gravity.
\end{abstract}

\section{Introduction}

$\mathrm{T}$ he absence of buoyant flow increases the residence time in microgravity fires causing substantial changes in flame structure and consequently changes the properties of the produced smoke. In particular, the increased residence time in the high temperature zone where the smoke is formed, can increase the opportunity for growth in the size of the smoke particulate. However, no measurements of the properties of smoke from microgravity fires had been made at the time when the detectors were designed for both the space shuttle and the ISS. This absence of data caused the designers to base the design on 1-g information and the current state of the art. As a result, the two detectors were designed using different detection technologies which have very different particle size sensitivities. The objective of this work was to make sufficient measurements of smoke from spacecraft fires to enable improved design of future detectors.

\section{A. Smoke Background}

Smoke is a general term that encompasses aerosol materials produced by a number of processes. In particular it can include unburned, recondensed, original polymer or pyrolysis products that can be either liquid or solid, hydrocarbon soot, condensed water vapor, and ash particles. Ash and soot particles dominate the smoke particulate in established flaming fires while unburned pyrolysis products and recondensed polymer fragments are produced by smoldering and pyrolysis in the early stage of fire growth. Given the constrained space on any spacecraft, the target

\footnotetext{
${ }^{1}$ Branch Chief, Combustion and Reacting Systems Branch, 21000 Brookpark Road, MS 77-5, Senior Member.

${ }^{2}$ Advanced Capabilities Project Office, 21000 Brookpark Road, MS 77-7, Associate Fellow.

${ }^{3}$ Aerospace Engineer, Combustion and Reacting Systems Branch, 21000 Brookpark Road, MS 77-5.

${ }^{4}$ Integrated Performance Assessment Group Leader, 100 Bureau Drive, Stop 8664.

${ }^{5}$ Fire Metrology Group Leader, 100 Bureau Drive, Stop 8662.

${ }^{6}$ Visiting Professor of Mechanical Engineering, Glenn L. Martin Hall.

${ }^{7}$ Staff Scientist, 21000 Brookpark Road, MS 77-5.
} 
for the fire detection system is necessarily the early phase and not established flaming fires; consequently, the primary target for detection is the pyrolysis products and not the soot and ash.

\section{B. Prior Spacecraft Systems}

In the Mercury, Gemini and Apollo missions, the crew quarters were limited and mission durations were short, consequently it was considered reasonable that the astronauts would rapidly detect any fire. The Skylab module, however, included approximately $30 \mathrm{UV}$-sensing fire detectors. ${ }^{1}$ These devices were limited to line-of-sight and were reported to have difficulties with false alarms.

\section{Space Shuttle Detectors}

At the time the Space Shuttle Orbiter (Space Transportation System (STS)) was developed, smoke detection for terrestrial applications was becoming commonplace. ${ }^{2}$ In particular, ionization detectors were becoming readily available but photoelectric (scattering or obscuration detectors) were generally unavailable due to the difficulty of producing stable light sources. There was no data available concerning the smoke or dust particle size distribution in low-gravity and the database of normalgravity smoke characteristics was a fraction of what is available today. Consequently, the STS smoke detector (Fig. 1) employed a variant of the accepted ground based approach (ionization detectors). The Orbiter has nine particle-ionization smoke detectors in avionics cooling air return lines in the mid-deck and flight deck and SpaceLab had six additional particle-ionization smoke detectors in the avionics lines. ${ }^{3}$ While the design rationale is not completely known, Celesco ${ }^{\mathrm{TM}}$ (later Brunswick Defense ${ }^{\mathrm{TM}}$ ) based their design on data that suggested incipient fires could be discriminated by looking for particulate in the 0.4 to $0.7 \mu \mathrm{m}$ range. ${ }^{4}$ Use of a pump provided the opportunity to employ a particle separator allowing rejection of particulate larger than $1 \mu \mathrm{m}$. This air pump increased the power requirements and reduced the operational life for the detectors.

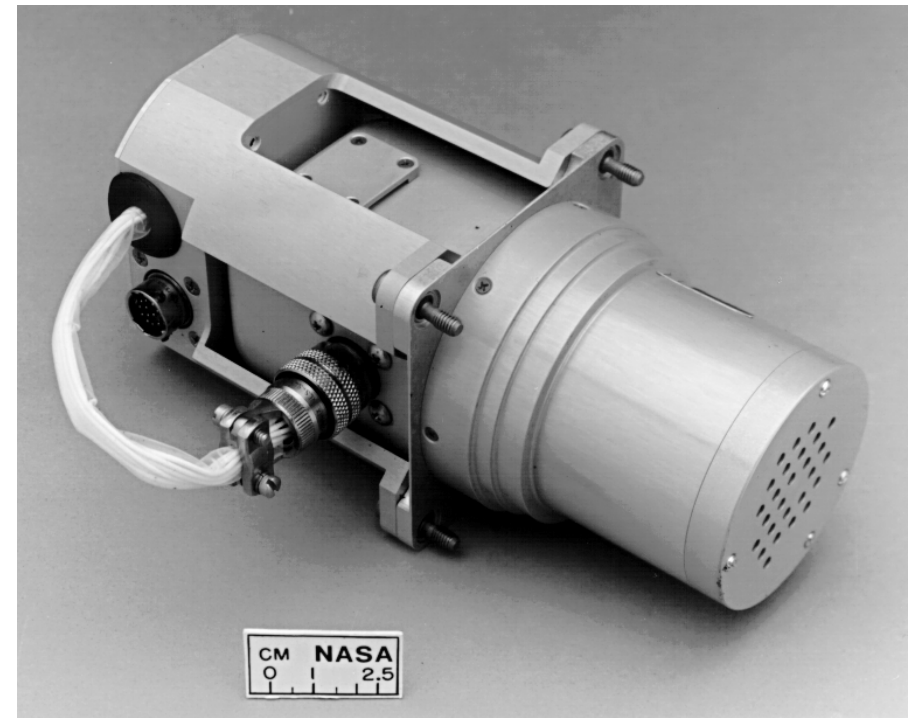

Figure 1. Brunswick Defense ${ }^{\mathrm{TM}}$ smoke detector used in the NASA Orbiter fleet. The inlet is on the right and the gas is expelled out the small plate on the top.

\section{ISS Detectors}

The International Space Station (ISS) smoke detector design took advantage of the availability of stable laserdiode light sources, mirroring a trend in terrestrial detectors toward light scattering devices. This provided the opportunity to produce a detector that required substantially less power, (1.5 Watts versus 9 Watts for the Orbiter detector $^{5}$ and, with no moving parts, was quieter with a much longer operational life. The choice was supported by data indicating that early smoldering fires produce larger particulate than established flaming fires. ${ }^{2}$ The designated detectors (Fig. 2) for the ISS are laser-diode, forward-scattering, smoke or particulate detectors. Their sensitivity is greatest for particles larger than $1 \mu \mathrm{m}$ with sensitivity extending down to $0.6 \mu \mathrm{m}$. The current requirements for the ISS call for two detectors in the open area of the US Lab module, and detectors in racks that have cooling air-flow. ${ }^{6}$

The design developed for the ISS by Allied Signal ${ }^{\mathrm{TM}}$ (Fig. 2) consists of a 2-pass laser diode to sense forward scattered light (30 degrees). There is also a zero degree obscuration system that is used as a measure of the beam strength. The system is designed to alarm based on the magnitude of the scattered light signal referenced to the beam intensity. The system is less sensitive to particles smaller than the wavelength of the laser (near IR) than it is to larger particles. The minimum reported sensitivity is $0.3 \mu \mathrm{m} .{ }^{5}$ On the other hand, for a fixed mass concentration of smoke aerosol; the STS ionization smoke detector is more sensitive to smaller particle sizes. 
As a result of these design decisions, the ISS and the Orbiter detectors are sensitive to particles in different size ranges. In both cases the designs were based upon the best available data however, due to the complete absence of low-g data concerning the nature of particulate and radiant emission from incipient and fully developed low-g fires, different conclusions were drawn concerning the optimal design for spacecraft fire detection.

As described by Friedman ${ }^{7}$ there have been six overheat and failed component failures in the NASA Orbiter fleet. None of these events spread into a real fire but as mission durations increase, the likelihood of failures increases. The experience on Mir in 1997 has shown that failure of oxygen generation systems can have significant consequences. As a result, improved understanding of spacecraft fire detection is critically needed. ${ }^{8}$

\section{E. Background in Low Gravity Smoke}

The salient result on smoke particle size determination in low-gravity reported by Urban et al. ${ }^{9}$ is that particulate produced by low-gravity flames (soot or unburned fuel particles) tends to have larger size particles than in normal gravity. Results from the CSD (Comparative Soot Diagnostics) Experiment ${ }^{9}$ which studied smoke properties in low-gravity from several spacecraft materials suggested that liquid smoke particles could achieve sizes larger than $1 \mu \mathrm{m}$ while solid particulate remained in the sub-micrometer range. The combined impact of these limited results and theoretical predictions is that, as opposed to extrapolation from 1-g data, direct knowledge of low-g combustion particulate is needed for more confident design of smoke detectors for spacecraft.

\section{F. Spacecraft Atmosphere Dust Background}

Any effort to detect fires must be able to discriminate against the ambient background and nuisance signals. In the case of smoke detection, the background dust aerosol conditions must be considered. The only spacecraft background aerosol particulate measurements to date were made on the Orbiter on STS 32 in $1990^{10}$ and on the ISS. ${ }^{9}$ The STS measurements included the results from two cascade impactors and a light scattering device. The two impactors reported a bimodal particle size distribution with $\sim 40 \%$ of the particles in each of the 2.5 to $10 \mu \mathrm{m}$ and $>100 \mu \mathrm{m}$ ranges. The other two ranges $<2.5 \mu \mathrm{m}$ and 10 to $100 \mu \mathrm{m}$ were very lightly populated. Each impactor sampled approximately $15 \mathrm{~m}^{3}$ of air over approximately 30 hours. These results showed substantially higher concentrations than typical indoor measurements (over a factor of 5 for the two most populated size ranges). These data were supported by the light scattering instrument which made 17 measurements in 12 locations, all of these reported mass concentrations ranging from 50 to $70 \mu \mathrm{g} / \mathrm{m}^{3}$. The ISS results of Urban et al. ${ }^{9}$ were a secondary result of an experiment to validate the operation of an aerosol particle counter in low-gravity. The measurements were made in the US Lab on Increment 10 and the particle count levels were at the noise level for the instrument (less than 5 particles/cc). The low particle levels in the $<2.5 \mu \mathrm{m}$ bin for the STS and the low overall numbers for the ISS suggest a zone of opportunity for spacecraft fire detection since typical normal gravity fires produce substantial particulate in this size range. ${ }^{2,11}$ However more complete particle size statistics are needed. 


\section{SAME Experiment}

Although the CSD experiment produced useful information concerning the likely size of spacecraft smoke particulate, the data set was not particularly large as the size statistics were obtained from TEM data and critical details were not obtained for the liquid droplet aerosols. To rectify this concern, another experiment (Smoke Aerosol Measurement Experiment: SAME) was developed. The SAME experiment sought to avoid the problems experienced by the CSD experiment by obtaining the particulate size statistics on-orbit with a reduced dependence upon sample return to Earth. This is a challenging endeavor because existing aerosol instrumentation is typically large, incompatible with spacecraft experiment constraints, and may require substantial sample return to Earth. As will be described below, an alternative approach was employed that used three discrete instruments to measure separate moments of the size distribution. When combined, these moments provide useful aggregate statistics of the size distribution. The measurements were made using smoke generated by overheated spacecraft materials in much the same manner as the CSD experiment however the sample temperature, flow field, and particle aging time were more rigorously controlled.

\section{A. Moment Method}

The approach used by the SAME experiment is termed the 'moment method' for convenience. ${ }^{12}$ As will be described below, the approach consists of measuring three moments of the size distribution (zeroth, first and third) and using the properties of the log-normal distribution to estimate the geometric mean diameter and the standard deviation.

The average particle size and an estimate of the width of the size distribution will be estimated from various moments of the size distribution. The number distribution, $\mathrm{f}_{\mathrm{N}}(\mathrm{D})$, is defined as

$$
f_{N}(D)=\frac{d N}{d D}
$$

where $\mathrm{dN}$ is the number of particles per $\mathrm{cm}^{3}$ with diameter between $\mathrm{D}$ and $\mathrm{D}+\mathrm{dD}$. The moments of interest consist of the number concentration, $\mathrm{M}_{0}$, the first moment $\mathrm{M}_{1}$, and the volume or mass concentration moment, $\mathrm{M}_{3}$.

$$
M_{i}=\int D^{i} f_{N}(D) d D \quad i=0,1,3
$$

The first moment which can also be thought of as the "diameter concentration" or integrated diameter per unit volume is approximately proportional to the ionization detector moment (signal). For particles in the Mie scattering regime, particles sizes from $0.3 \lambda$ to about $3 \lambda(\sim 0.2$ to $2.0 \mu \mathrm{m}$ for a red laser $)$ the light scattering signal is approximately proportional to the third moment. From these moments, and a measurement of $\mathrm{M}_{0}$ using a condensation nucleus counter, two mean diameters can be computed: the count (arithmetic) mean diameter $\mathrm{D}_{0.5}$ or $\bar{d}$, which is equal to $\mathrm{M}_{1} / \mathrm{M}_{0}$ and the diameter of average mass $\mathrm{D}_{1.5}$ or $d_{\bar{m}}$, which is equal to $\left(\mathrm{M}_{3} / \mathrm{M}_{0}\right)^{1 / 3}$. The lognormal size distribution is widely used for describing the size distribution of aerosols including non-flaming smoke. As in most aerosols; the bulk of the number concentration is associated with smaller particles. ${ }^{13,14}$ The form of the distribution is the same as the normal distribution except that the diameter is replaced with the $\ln \mathrm{D}$ so that one has

$$
f_{N}(D)=\frac{N_{t}}{(2 \pi)^{1 / 2} D \ln \sigma_{g}} \exp \left(-\frac{\ln D-\ln D_{g}}{2 \ln ^{2} \sigma_{g}}\right)
$$

where $\mathrm{N}_{\mathrm{t}}$ is the total number concentration of the aerosol $\left(=\mathrm{M}_{0}\right)$, and $\mathrm{D}_{\mathrm{g}}$ and $\sigma_{\mathrm{g}}$ are the geometric mean number diameter and geometric standard deviation defined by

$$
\ln D_{g}=\int_{0}^{\infty} \ln D f_{N}(D) d D / \int_{0}^{\infty} f_{N} d D
$$




$$
\ln \sigma_{g}=\left[\int_{0}^{\infty}\left(\ln D-\ln D_{g}\right)^{2} f_{N}(D) d D / \int_{0}^{\infty} f_{N}(D) d D\right]^{1 / 2}
$$

For the log-normal distribution, one finds that the various diameter definitions are related to the geometric mean number diameter, $\mathrm{D}_{\mathrm{g}}$, via the equation: ${ }^{13,14}$

$$
D_{p}=D_{g} \exp \left(p \ln ^{2} \sigma_{g}\right)
$$

For the count mean diameter, $\mathrm{D}_{0.5}$, and the diameter of average mass, $\mathrm{D}_{1.5}$, the corresponding values of $\mathrm{p}$ are 0.5 and 1.5. As an example, for a log-normal distribution with $\mathrm{D}_{\mathrm{g}}=1.0 \mu \mathrm{m}$ and $\sigma_{\mathrm{g}}=2.0$, the corresponding values of $\mathrm{D}_{0.5}$ and $\mathrm{D}_{1.5}$ are $1.27 \mu \mathrm{m}$ and $2.06 \mu \mathrm{m}$, respectively. Using equation (6), one can relate $\sigma_{\mathrm{g}}$ to the ratio of $\mathrm{D}_{1.5}$ and $\mathrm{D}_{0.5}$ via the equation:

$$
\sigma_{g}=\exp \left(\ln \left(D_{15} / D_{0.5}\right)\right)^{1 / 2}
$$

By combining these three moments it is possible to compute three mean diameters of the size distribution and the geometric standard deviation. Validation of this approach is discussed in Cleary, Weinert and Mulholland. ${ }^{3}$ These statistics provide a strong basis for design of spacecraft smoke detectors.

\section{B. Instruments}

These measurements were made using an assembly of three separate instruments. Two are industrial hygiene instruments manufactured by TSI and one is a modified residential smoke detector.

The zeroth-moment instrument is a condensation nuclei counter P-Trak ${ }^{\mathrm{TM}}$ (TSI Inc.). This device operates by passing the aerosol-laden particle stream through a region where it is saturated with isopropanol vapor and then into a cooler region where the vapor condenses onto the particles raising them all to a size where they are readily counted by a light scattering device. This instrument is very robust and operates over a range of 0 to $105 \mathrm{particles} / \mathrm{cm}^{3}$ and $20 \mathrm{~nm}$ to $1 \mu \mathrm{m}$ diameter. Some dilution is required since the smoke concentration ranges from about $0.5 \times 10^{6}$ to 5 $\times 10^{6}$. There was also a concern that the isopropanol condensate would not return to the wick in low-gravity. ${ }^{9}$ To mitigate this issue, the condensing section of the device was modified with very small grooves to improve conductance of the condensate back to the wick. These changes were tested in a separate space experiment with good results indicating the modified device could be used successfully in low gravity. ${ }^{9}$

The first-moment instrument is the ionization chamber from a residential smoke detector which uses an alphaparticle emitter to generate ions in a region inside a DC electric field which causes the ions to drift producing current. The presence of particulate reduces the current as a result of the attachment of the ions to the particulate. The mobility of the charged aerosol is too small for it to be collected on the ionization chamber electrode. The required particle concentrations are on the order of 105 particles $/ \mathrm{cm}^{3}$ and no sample dilution was required.

The third-moment instrument is a light scattering device DustTrak ${ }^{\mathrm{TM}}$ (TSI Inc.). The device uses a 90 degree light scattering signal to quantify the aerosol mass density. For terrestrial dust particulate this signal correlates well with the mass concentration, however additional compensation will be needed to account for the range of particle sizes that will be seen in the SAME experiment. The device operating range is from 0.001 to $100 \mathrm{mg} / \mathrm{m}^{3}$. These devices are equipped with an aerodynamic impactor at the inlet which captures particles larger than the selected size. The SAME experiment included 2 DustTraks $^{\mathrm{TM}}$ one with a $1 \mu \mathrm{m}$ impactor and one with a $10 \mu \mathrm{m}$ impactor. The difference in the signal from these two devices provided a measure of the fraction of the particulate that was larger than $1 \mu \mathrm{m}$. In some cases dilution was required owing to the high smoke concentration levels.

A schematic of the assembled hardware appears in Fig. 3. Smoke was generated by overheating a small sample of material in the smoke generation duct for approximately 60 seconds. During this interval, controlled flow was induced by a moving piston in the aging chamber which drew the smoke into the chamber. The smoke was held in the chamber for a predetermined time, allowing the particles to coagulate. The smoke was then pushed by the piston into the diagnostics duct where the moment instruments made their measurements. Also installed in the diagnostics duct were space shuttle and ISS smoke detectors. As the smoke was monitored by the moment instruments, a 
sample of the smoke particles was deposited on Transmission Electron Microscope (TEM) grid via a thermal precipitator which uses thermophoresis to deposit the particles on the grids.

\section{Experimental Results}

The system was installed in the Microgravity Science Glovebox, an ISS facility that provides many resources including: containment, power, data, video and uplink commanding. The assembled hardware is shown in Fig 4. Overall 30 sample materials were tested. These were comprised of six samples each of 5

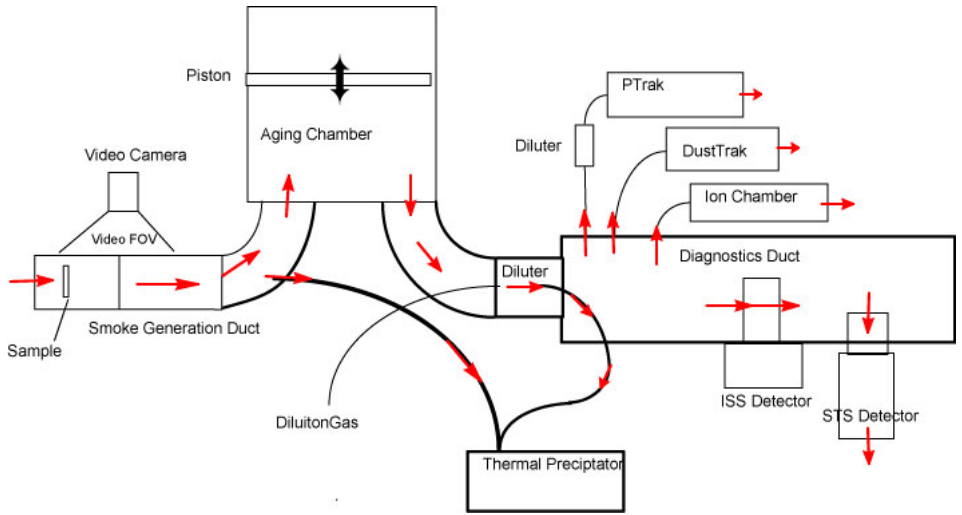

Figure 3. Schematic of the SAME hardware. materials: Teflon ${ }^{\mathrm{TM}}$, Kapton ${ }^{\mathrm{TM}}$, silicone rubber, cellulose, and dibutyl-phthalate deposited on a porous wick. These samples were tested at different airflow rates, pyrolysis temperatures, and smoke aging durations. The analog signals from the diagnostics were digitized at 1 Hz. At this time the used samples and TEM grids have just been received back from the ISS so mass-balances and TEM analysis have not been initiated. The zeroth and first moment instruments have a limited dependence of particle diameter so they may be directly calibrated. The third moment instrument (the DustTrak $^{\mathrm{TM}}$ ) has a significant dependence on the particle diameter and the refractive index. An algorithm has been implemented to use the results of a Mie Scattering calculation with the measured results to iteratively converge on the value of the third moment that best fits the DustTrak $^{\mathrm{TM}}$ signal and the other two moment measurements. This work is currently underway so only results from the zeroth and

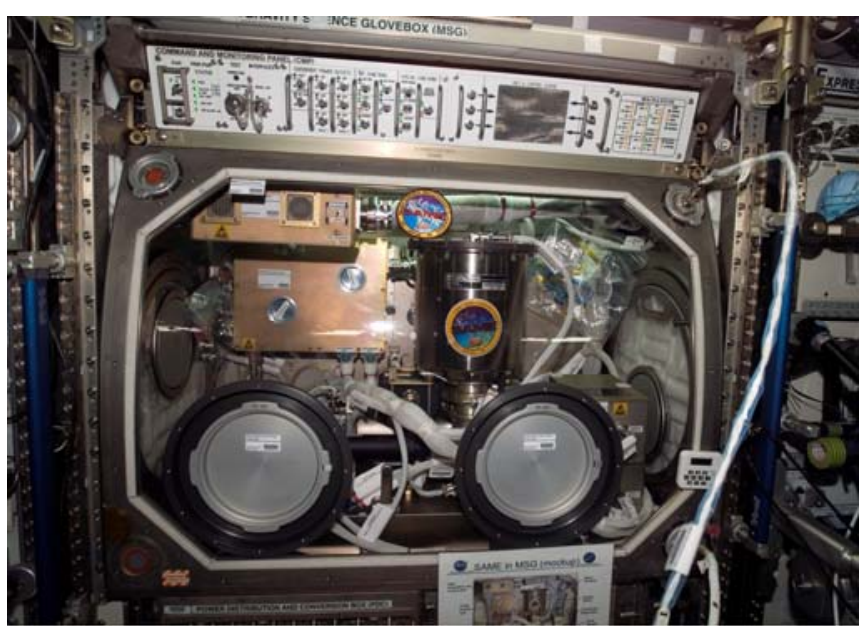

Figure 4. SAME Experiment installed in the MSG Facility first moment instruments will be presented here. As discussed above, the ratio of the first to the zeroth moment is the count mean diameter $\left(\mathrm{D}_{0.5}\right)$. Values of $\mathrm{D}_{0.5}$ were calculated directly from the calibrated signals from the P-Trak and the Ion sensor and are shown in Table 1 for the baseline runs of the 4 solid materials with the percentage increase over the sizes from a comparable test in normal gravity.

\section{Smoke Transport Model}

To assist in the interpretation of the SAME results and to provide a further understanding of the implementation of smoke detectors in spacecraft applications, a numerical model of the smoke transport and detection in the Destiny Laboratory (US Lab) of the ISS was developed. The details of the model are discussed by Brooker et al. ${ }^{15}$ This study was conducted using FDS (Fire Dynamics Simulator) a fire modeling program developed by McGrattan et al. ${ }^{16,17}$ at NIST. FDS (version 4.0.7 Linux) is a computational fluid dynamics (CFD) model that numerically solves a form of the Navier-Stokes equations for low speed, thermally driven flow with an emphasis on smoke and heat transport from fires. ${ }^{16}$ A detailed description of the theoretical equations, underlying

\begin{tabular}{|l|c|c|}
\hline \multicolumn{1}{|c|}{ Material } & $\begin{array}{c}\text { Count } \\
\text { Mean } \\
\text { Diameter (nm) }\end{array}$ & $\begin{array}{c}\text { Count } \\
\text { Mean Diameter } \\
\text { (\% larger than } \\
\text { 1-g) }\end{array}$ \\
\hline $\begin{array}{l}\text { Silicone } \\
\text { Rubber }\end{array}$ & 263 & 53 \\
\hline Teflon $^{\mathrm{TM}}$ & 223 & \\
\hline Lamp wick $^{\text {Mamp }}$ & 248 & 65 \\
\hline Kapton $^{\mathrm{TM}}$ & 114 & 58 \\
\hline
\end{tabular}

Table 1: Count Mean Diameters for Baseline Runs with comparison to 1-g tests. 
assumptions, and numerical solution can be found in the technical reference guide. ${ }^{17}$ For this application, large eddy simulation (LES) was utilized in which the large scale eddies are computed directly and the sub-grid scale dissipative processes are modeled. Since the ISS is in low earth orbit, the gravitational acceleration was set to zero. As a result, the vorticity generated by buoyancy is no longer present in the momentum equation; therefore, the baroclinic torque was included to account for other sources of vorticity. A mixture fraction combustion model (a conserved quantity representing the fraction of material at a given point that originated in the fuel stream) was utilized for this analysis. All solid surfaces were designated as inert with a thermal boundary condition of $293 \mathrm{~K}$. The number of grid cells spanning each coordinate direction was determined such that the cell dimension was approximately $5 \mathrm{~cm}$ in length for a total of 259,200 cells. The model layout is shown in Fig 5.

The smoke generation was modeled using a source positioned at the center of each rack location or ISPR (International Standard Payload Rack). The source was modeled as a propane burner with an area of $0.023 \mathrm{~m}^{2}$ and heat flux of 57.6 $\mathrm{kW} / \mathrm{m}^{2}$ at three soot yield levels, producing smoke particulate outputs of 1.46, 2.95, and 8.47

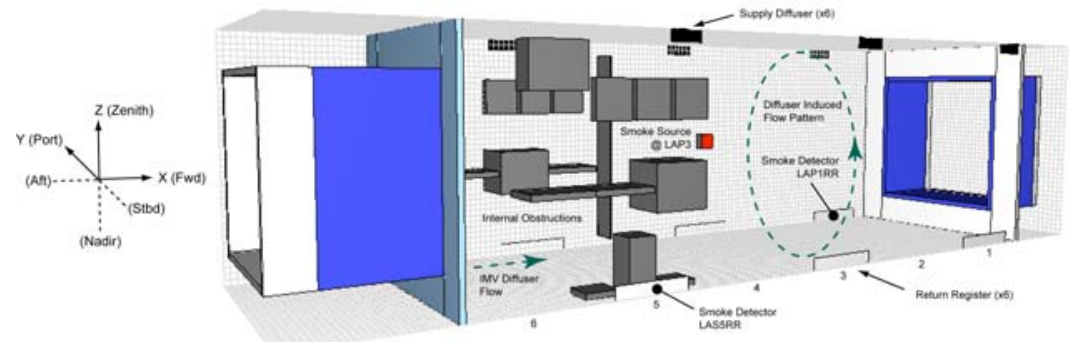

Figure 5. Computational Model Domain showing six overhead supply diffusers, six deck return registers, and one aft-port IMV diffuser for air ventilation. Induced flow patterns are indicated with dashed lines. Two area smoke detectors are located in front of LAP1 return register (LAP1RR) and LAS5 return register (LAS5RR). Also shown are internal obstructions representing on-orbit equipment and a smoke source at LAP3. ISPR sections are numbered one through six (fwd to aft) and labeled: port (P), starboard (S), nadir (N), or zenith (Z). $\mathrm{mg} / \mathrm{s}$. The alarm point for the ISS detector is on the order of $0.5 \mathrm{mg} / \mathrm{m}^{3}$. The computational model was run at each smoke generation rate and rack location. The flow conditions in the lab were established to duplicate the operation of the ventilation system on orbit. The return and diffuser vanes are oriented to create an overall toroidal flow field that rotates counterclockwise (looking forward). Axial flow is induced near the floor on the port side by the Inter Module Ventilation (IMV) register on the aft bulkhead. For smoke generation locations at sections 5 and 6 , the simulations were also run with and without the internal obstructions shown in Fig. 5. These internal obstructions were positioned to simulate typical installed hardware in the US Lab Module. The smoke source was

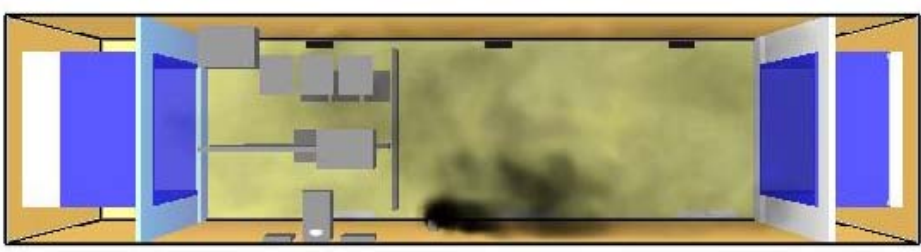

Figure 6: Representation of the smoke distribution 177 seconds after the smoke source was activated at location 4 on the floor. The smoke generation rate was $8.5 \mathrm{mg} / \mathrm{s}$.

activated 30 seconds after the simulation was initiated and the simulations were run for an additional 300 seconds. Figure 6 presents a reconstruction of the smoke distribution for an $8.5 \mathrm{mg} / \mathrm{s}$ generation rate case after 177 seconds. It is notable that other than the high concentration zone near the source, the smoke is fairly well mixed throughout the region without obstructions. The region with obstructions has lower smoke levels than the rest of the module. This scenario is substantially different from that seen in normal gravity where the smoke concentrates near the ceiling and mixing is generally not as complete as seen in Fig. 6.

Figures 7, 8 and 9 show the alarm time response for sources at Section LA1 (Fig. 7), LA3 (Fig. 8) and LA6 (Fig.9). Evident in the figures is the variation in detection times with soot yield and source location. Although for LA3, for the zenith smoke source, the detectors alarmed at the same time, overall one detector typically alarmed significantly sooner than the other, particularly at low smoke yield levels. For LA1and LA3, the port detector was the first to alarm in most cases while by LA6 the starboard detector was the first to alarm. This behavior is consistent with the relative proximity of the detectors to the smoke sources. In Fig. 9, the impact of the obstructions is evident. For sources on the floor, the obstruction had no effect. This is consistent with expectation since the 
floors are unobstructed and the flow is across the floor toward the detector. For cases on the starboard or zenith, the obstructions had a strong impact due to the longer transit time of the smoke to the detector.
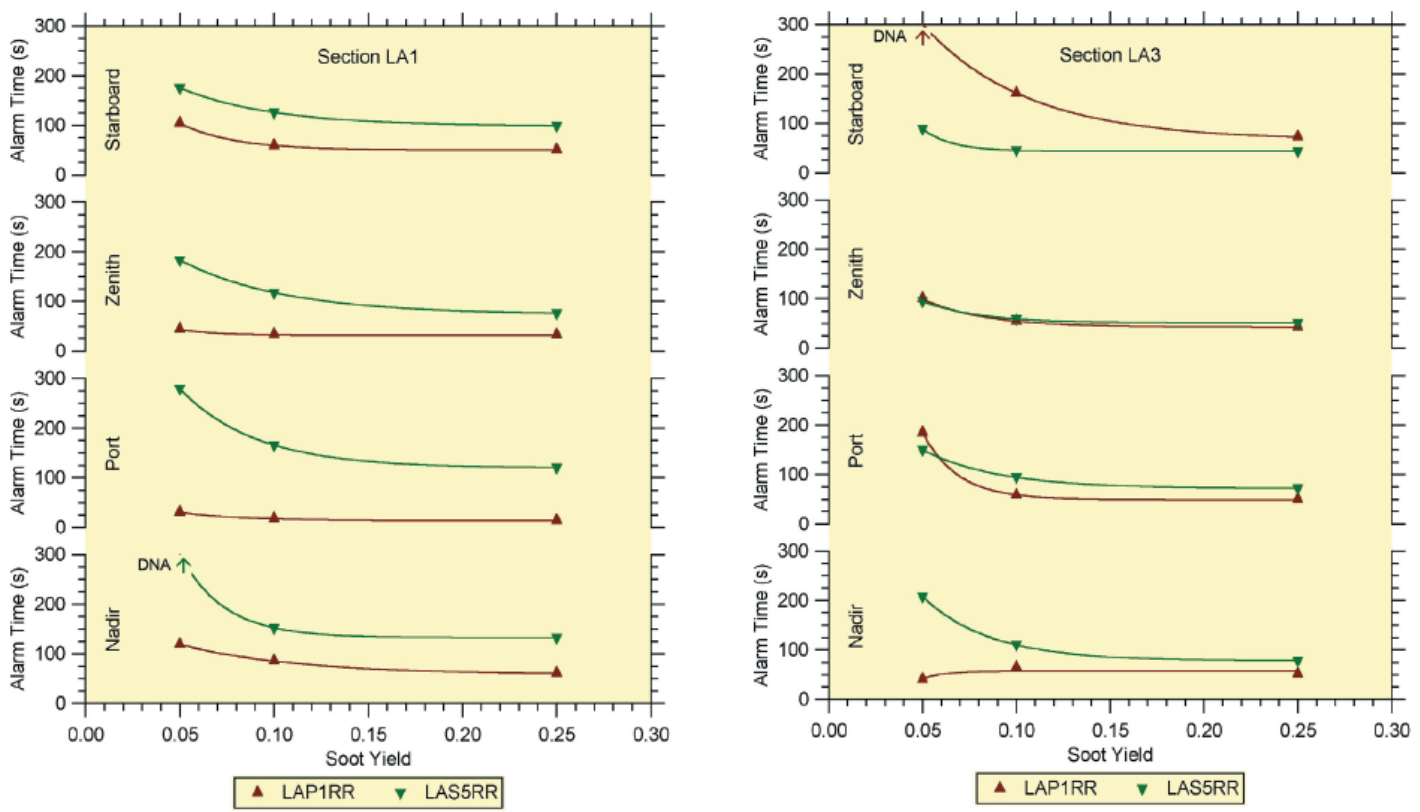

Figure 7 (left) and 8 (right): Smoke detector alarm time response to a variable strength smoke source. Data is from a circumferential profile at section LA1 (Fig. 7) and LA3 (Fig. 8). Note that a DNA (did not alarm) condition occurred for LA1 for the 0.05 soot yield on the N1 wall for LAS5RR and for LA3 0.05 soot yield on the S3 wall for LAP1RR.

\section{Conclusion}

Smoke particulate produced in low-gravity was found to be typically $50 \%$ large in count mean diameter than similar conditions in normal gravity. The resultant sizes were all below $300 \mathrm{~nm}$ suggesting that discriminating smoke from spacecraft dust possibly could be achieved by detecting in the sub-micrometer range. Numerical modeling of smoke transport in the US Lab Module of the ISS predicted that actual detection times can be quite long and strongly dependent on location and the presence of obstructions.

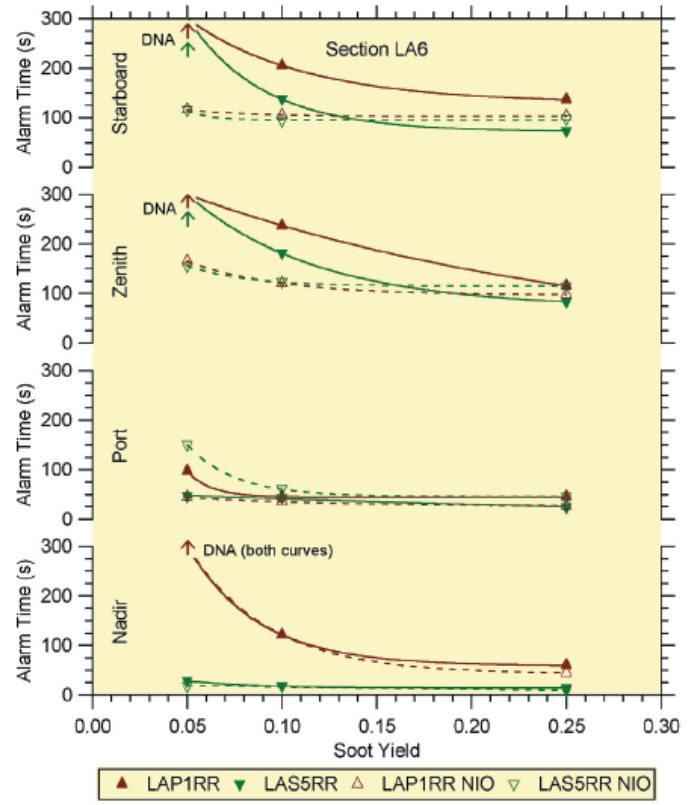

Figure 9. Smoke detector alarm time response to a variable strength smoke source. Data is from a circumferential profile at section LA6. Note that a DNA (did not alarm) condition occurred for the 0.05 soot yield on the S6, Z6, and N6 walls for LAP1RR, and S6 and Z6 walls for LAS5RR. Solid lines and symbols: with internal obstructions, dashed lines and open symbols: no obstructions. 


\section{Acknowledgments}

The support of the SAME experiment team and the crews of ISS increments 10, 13, and 15 are gratefully acknowledged. The SAME project was conducted through the ISS Exploration Research Project of the Exploration Technology Development Program. The support of this program is also acknowledged.

Certain commercial entities, equipment, or materials may be identified in this document in order to describe an experimental procedure or concept adequately. Such identification is not intended to imply recommendation or endorsement by the National Aeronautics and Space Administration or the National Institute of Standards and Technology, nor is it intended to imply that the entities, materials, or equipment are necessarily the best available for the purpose.

\section{References}

${ }^{1}$ Friedman, R., "Fire Safety Practices and Needs in Human-Crew Spacecraft," Journal of Applied Fire Science, Vol. 2, 1992, pp. 243-259.

${ }^{2}$ Bukowski, R.W. and G.W. Mulholland, "Smoke Detector Design and Smoke Properties,” NBS Technical Note $973,1978$.

${ }^{3}$ Martin, C.E. and R.C. DaLee, "Spacecraft Fire Detection and Suppression (FDS) Systems: An Overview and Recommendations for Future Flights," SAE Technical Paper 932166, 23rd International Conference on Environmental Systems, Colorado Springs, CO, July 12-15, 1993.

${ }^{4}$ National Research Council, Fire Detection for Life Safety, National Academy of Sciences, Washington D.C., 1975.

${ }^{5}$ Steisslinger, H. R., D. M. Hoy, J. A. McLin and E. C. Thomas, SAE Paper 932291, 19th International Conference on Environmental System, 1993.

${ }^{6}$ McKinnie, J., "Fire Response Aboard the International Space Station," SAE Technical Paper 972334, 27th International Conference on Environmental Systems, Lake Tahoe, NV, July 14-17, 1997.

${ }^{7}$ Friedman, R., "Risks and Issues in Fire Safety on the Space Station," NASA TM 106430, 1994.

${ }^{8}$ Ruff, G.A., D.L. Urban, and M.K. King, "A Research Plan for Fire Prevention Detection and Suppression," AIAA-20050341, 43 ${ }^{\text {rd }}$ Aerospace Sciences Meeting and Exhibit, Reno, NV, January 9-12, 2005.

${ }^{9}$ Urban, D.L., Griffin D., Ruff, G.A., Cleary, T., Yang, J., Mulholland, G., Yuan, Z.G., "Detection of Smoke from Microgravity Fires”, 2005 International Conference on Environmental Systems, Rome Italy, Paper \# 2005-01-2930 July 2005, SAE Transactions, pp 375-384.

${ }^{10}$ Liu, B. Y. H., K. L. Rubow, P. H. McMurry, T. J. Kotz and D. Russo, “Airborne Particulate Matter and Spacecraft Internal Environments," SAE Technical Paper 911476, 21st International Conference on Environmental Systems, San Francisco, CA , July 15-18, 1991.

${ }^{11}$ Bukowski, R. W, R. D. Peacock, J. D. Averill, T. G. Cleary, N. P. Bryner, W. D. Walton, P. A. Reneke, and E. D. Kuligowski, "Performance of Home Smoke Alarms, Analysis of the Response of Several Available Technologies in Residential Fire Settings," NIST Technical Note 1455, December 2003.

${ }^{12}$ Cleary, T.G., D W. Weinert, and G.W. Mulholland, "Moment Method for Obtaining Particle Size Measures of Test Smokes", Natl. Inst. Stand. Technol., NISTIR 7050, 2003.

${ }^{13}$ Raabe, O.G., J. Aerosol Sci., Vol. 2, 1971, p. 289.

${ }^{14}$ Reist, P.C., Introduction to Aerosol Science, Macmillan Pub. Co., NY, NY, 1984.

${ }^{15}$ Brooker, J. E., Urban, D.L., Ruff, G.A., "ISS Destiny Laboratory Smoke Detection Model”, 07ICES-113, 2007 International Conference on Environmental Systems, Chicago, Ill. July 2007.

${ }^{16}$ K.B. McGrattan and G.P. Forney. "Fire Dynamics Simulator (Version 4) User Guide". NIST Special Publication 1019, National Institute of Standards and Technology, Gaithersburg, Maryland, March 2006.

${ }^{17}$ K.B. McGrattan (editor). "Fire Dynamics Simulator (Version 4) Technical Reference Guide". NIST Special Publication 1018, National Institute of Standards and Technology, Gaithersburg, Maryland, March 2006. 